\title{
Imaging characteristics of chronic spinal cord injury identified during screening for a cell transplantation clinical trial
}

\author{
Joshua D. Burks, MD, ${ }^{1}$ Katie L. Gant, PhD, ${ }^{1,2}$ James D. Guest, MD, PhD, ${ }^{1,2}$ Aria G. Jamshidi, MD, ${ }^{1}$ \\ Efrem M. Cox, MD, ${ }^{1}$ Kim D. Anderson, PhD, ${ }^{1,2}$ W. Dalton Dietrich, $\mathrm{PhD},{ }^{1,2}$ \\ Mary Bartlett Bunge, PhD, ${ }^{1-3}$ Barth A. Green, MD, ${ }^{1,2}$ Aisha Khan, MBA, ${ }^{4}$ Damien D. Pearse, PhD, ${ }^{1,2}$ \\ Efrat Saraf-Lavi, MD, ${ }^{5}$ and Allan D. Levi, MD, $\mathrm{PhD}^{1,2}$ \\ Departments of ${ }^{1}$ Neurological Surgery, ${ }^{3} \mathrm{Cell}$ Biology, and ${ }^{5}$ Radiology; ${ }^{2}$ The Miami Project to Cure Paralysis; and ${ }^{4}$ The \\ Interdisciplinary Stem Cell Institute, The University of Miami Miller School of Medicine, Miami, Florida
}

OBJECTIVE In cell transplantation trials for spinal cord injury (SCI), quantifiable imaging criteria that serve as inclusion criteria are important in trial design. The authors' institutional experience has demonstrated an overall high rate of screen failures. The authors examined the causes for trial exclusion in a phase I, open-lab clinical trial examining the role of autologous Schwann cell intramedullary transplantation. Specifically, they reviewed the imaging characteristics in people with chronic SCl that excluded applicants from the trial, as this was a common cause of screening failures in their study. METHODS The authors reviewed MRI records from 152 people with chronic (> 1 year) SCl who volunteered for intralesional Schwann cell transplantation but were deemed ineligible by prospectively defined criteria. Rostral-caudal injury lesion length was measured along the long axis of the spinal cord in the sagittal plane on T2-weighted MRI. Other lesion characteristics, specifically those pertaining to lesion cavity structure resulting in trial exclusion, were recorded.

RESULTS Imaging records from 152 potential participants with chronic SCl were reviewed, 42 with thoracic-level SCI and 110 with cervical-level SCl. Twenty-three individuals (55\%) with thoracic SCI and $70(64 \%)$ with cervical SCI were not enrolled in the trial based on imaging characteristics. For potential participants with thoracic injuries who did not meet the screening criteria for enrollment, the average rostral-caudal sagittal lesion length was $50 \mathrm{~mm}$ (SD $41 \mathrm{~mm}$ ). In applicants with cervical injuries who did not meet the screening criteria for enrollment, the average sagittal lesion length was $34 \mathrm{~mm}$ (SD $21 \mathrm{~mm})$.

CONCLUSIONS While screening people with SCI for participation in a cell transplantation clinical trial, lesion length or volume can exclude potential subjects who appear appropriate candidates based on neurological eligibility criteria. In planning future cell-based therapy trials, the limitations incurred by lesion size should be considered early due to the screening burden and impact on candidate selection.

https://thejns.org/doi/abs/10.3171/2018.12.FOCUS18593

KEYWORDS spinal cord injury; cell transplantation; Schwann cell; screening; yield; enrollment

$\mathrm{T}$ HE annual estimated cost attributable to spinal cord injury (SCI) is approximately $\$ 14.5$ billion, ${ }^{16}$ and the emotional burden on affected individuals and their families is immeasurable. There are as many as 270,000 people living in the United States with SCI. ${ }^{19}$ Although some spontaneous recovery occurs in the early period fol- lowing SCI, limited spontaneous neurological improvement is expected in the chronic phase of the disease course ( $\geq 12$ months postinjury). Cellular transplantation strategies have been systematically evaluated for many years in animal models, with evidence of improved neurological recovery. ${ }^{3,17,18}$ The FDA has approved a few clinical tri- 
als designed to evaluate the safety of cellular transplantation in people with SCI (NCT02354625, NCT02302157, NCT01321333, and NCT01772810), 1,6,10,15

In order to establish safety, eligibility criteria are necessarily strict for exploratory cell transplant trials in SCI, limiting enrollment despite great interest from the SCI community. Inclusion and exclusion criteria typically include standard demographic information and SCI characteristics, including age, time since injury, cause of injury, neurological level of injury, and injury grade, as determined by the International Standards of Neurological Classification of Spinal Cord Injury developed by the American Spinal Injury Association, ${ }^{12}$ as well as other relevant body systems information. For clinical trials involving cell transplantation, additional eligibility criteria related to definable features of the injury site within the spinal cord are considered during the screening process.

Currently, MRI is the gold standard in assessing the morphology and anatomical integrity of the spinal cord after SCI. ${ }^{4}$ MRI is usually performed acutely in the initial assessment of a suspected SCI and can provide information about the severity and extent of injury, which may offer some prognostic value in the prediction of functional recovery. ${ }^{9}$ In people with chronic SCI ( $>1$ year postinjury), imaging of the spinal cord is rarely performed unless a decline in neurological function or a significant increase in pain is reported. For this reason, there are few systematic studies regarding the features of chronic SCI as evaluated by MRI.

One of the earliest published reports detailing radiological findings in chronic SCI evaluated MRI studies of people who were experiencing new pain or worsening of neurological function. ${ }^{21}$ T2-weighted spin-echo pulse sequences with long echo times were noted to most accurately demonstrate intramedullary abnormalities in the spinal cord, which aided in the diagnosis and treatment of posttraumatic spinal cord cysts. Later MRI studies sought to characterize chronic SCI in larger samples, including people with stable neurological function. . $^{2,23,28}$

The most extensive study to date was completed by Wang et al., ${ }^{27}$ in which 153 people with chronic SCI were assessed neurologically and radiologically. In this study, all participants had sustained SCIs more than 20 years prior to enrollment, ranging from 21 to 48 years. Patterns of pathology within the spinal cord were characterized and included extended atrophy, myelomalacia, syrinx, cyst, cord disruption, and tethering. No clear correlation was identified between any of the pathologies and the age at the time of imaging or time since injury.

Studies involving the postmortem evaluation of the spinal cord ${ }^{20,24}$ have added value to our understanding of the structural characteristics of chronic SCI. Squier and Lehr ${ }^{24}$ analyzed spinal cords of 20 people with SCI and found that $20 \%$ had cystic cavities extending at least 2 segments from the site of injury, similar to what was reported in imaging studies. ${ }^{22} \mathrm{~A}$ similar postmortem analysis of 50 spinal cords by Quencer and Bunge ${ }^{20}$ made an important distinction between expanding posttraumatic intramedullary cysts (syrinxes) and nonexpanding cavitation (cysts). At that time, they identified cystic areas as a potential target for cellular transplantation.
With early-phase cell transplantation clinical trials in chronic SCI currently underway and more on the horizon, in addition to future efficacy trials, it is important for research teams to consider typical radiological features. Whether cells are delivered intra- or perilesionally, intramedullary architecture will most certainly be considered during the screening process. A major challenge in moving ahead with clinical trials involving cellular transplantation in people with SCI is identifying eligible study participants. Thus far, strict inclusion and exclusion criteria have substantially limited the number of candidates selected during the screening phase. For example, the Schwann cell clinical trial in chronic SCI (NCT02354625) being conducted at The Miami Project to Cure Paralysis prescreened over 400 interested applicants in order to enroll only 8 study participants. Given the cost and resources required to initiate such a trial, we are describing our experience of screening potential participants with chronic SCI. Since the majority of applicants failed the screening process due to characteristics identified by radiographic evaluation, we report here the imaging characteristics of SCI based on MRI evaluation.

We examined the imaging characteristics of SCI in people excluded from our own institution's clinical trial (NCT02354625) and characterized features on MRI that were associated with exclusion, as this made up the majority of prescreen failures. As interest in cell transplantation for SCI continues to grow, it is important to refine the screening process. No previous studies have clarified the radiological features of chronic SCI that do not meet current criteria for such trials. Our aim is to better understand characteristics and imaging features that necessitate exclusion for proper allocation of resources in future trials.

\section{Methods}

People with chronic SCI (> 1 year postinjury) were invited to prescreen for the Schwann cell clinical trial (NCT02354625), conducted by The Miami Project to Cure Paralysis, a center of excellence at the University of Miami Miller School of Medicine. Screening information was provided in person, over the phone, or by email and included basic demographic and SCI details. In order to further determine eligibility, applicants were asked to submit an MRI of the spinal cord (from at least 1 year postinjury), among other documentation requested. Although over 400 people provided information for the initial screening process, only 160 subsequently submitted MRIs to the research team. Eight of those people were eventually enrolled in the trial, following further screening including a repeat MRI scan, and the other 152 were deemed ineligible based on established inclusion and exclusion criteria. The reason for exclusion from the trial was documented for each prescreened individual.

Eligibility criteria related to imaging were as follows:

1) Inclusion: total lesion length $\leq 3 \mathrm{~cm}$ and lesion volume $\leq 2 \mathrm{~cm}^{3}$, as approximated on MRI. These parameters were established based on safety data generated during our previous study of Schwann cell transplantation in people with subacute SCI. ${ }^{1}$ That dose-escalation study examined transplantation of 5 
TABLE 1. Participant characteristics

\begin{tabular}{lc}
\hline \multicolumn{2}{c}{ Characteristic } \\
\hline Sex, no. (\%) \\
\hline Male & $118(78)$ \\
\hline Female & $34(22)$ \\
\hline Mean age at imaging, yrs (SD) & $36(15)$ \\
\hline Mean age at injury, yrs (SD) & $28(10)$ \\
\hline Neurological level of injury, no. (\%) & $110(72)$ \\
\hline Cervical & $42(28)$ \\
\hline Thoracic & $90(59)$ \\
\hline Cause of injury, no. (\%) & $11(7)$ \\
\hline MVA & $20(13)$ \\
\hline Diving & $27(18)$ \\
\hline Fall & $4(3)$ \\
\hline Sports
\end{tabular}

million, 10 million, and 15 million Schwann cells ( $\mathrm{n}=2$ in each cohort). For this trial in chronic SCI, a cavity-filling dose up to 200 million cells in $2 \mathrm{~cm}^{3}$ was approved.

2) Exclusion: persons unable to safely undergo an MRI.

3) Exclusion: persons with penetrating injury of the spinal cord or complete transection of the cord, as identified on MRI.

4) Exclusion: persons with severe, uncorrected postinjury spinal deformity and/or a spinal cord inadequately decompressed.

5) Exclusion: persons with a cavity structure that would preclude successful transplantation, as identified on MRI, which may include septations or irregularities in tissue structure.

6) Exclusion: persons with syringomyelia, defined as those with progressively enlarging cysts on T2weighted images with associated neurological decline.

The study investigators reviewed T2-weighted sagittal images, and the following aspects of the SCI were characterized and measured: A) injury lesion length: rostral-caudal extent of signal abnormality; B) incidence of cyst:23,27 a well-defined area of hyperintense T2 within the spinal cord; C) incidence of myelomalacia: ${ }^{23,27}$ an area of less hyperintense signal intensity (between CSF and normal spinal cord) within the spinal cord with more poorly defined margins and irregular shape; D) incidence of tethering: attachment of the spinal cord to the wall of the spinal canal; and E) incidence of septations: ${ }^{27}$ irregularities or divisions within a cyst or area of myelomalacia.

Categorical variables with percentages and continuous variables are reported as means with SDs. Statistical analysis was performed using SPSS software (version 22, IBM Corp.). This study was performed in accordance with the University of Miami Institutional Review Board.

\section{Results}

MRI studies from a total of 152 people with chronic
TABLE 2. Reasons for prescreening failure by imaging criteria

\begin{tabular}{lcc}
\hline \multirow{2}{*}{ Variable } & \multicolumn{2}{c}{ Level of Injury (\%) } \\
\cline { 2 - 3 } & Cervical & Thoracic \\
\hline Total & $70 / 110(64)$ & $23 / 42(55)$ \\
\hline Rostral-caudal length $>3 \mathrm{~cm}$ & $53(48)$ & $10(24)$ \\
\hline Cavity structure & $16(15)$ & $12(29)$ \\
\hline Syringomyelia & $1(1)$ & $1(2)$ \\
\hline
\end{tabular}

SCI who submitted screening documentation for the trial were assessed. Of these, $118(78 \%)$ were male and 34 $(22 \%)$ were female. The mean age at imaging was 36 years (SD 15 years), and the mean age at injury was 28 years (SD 10 years). Ninety people (59\%) had injuries related to a motor vehicle accident (MVA), 11 (7\%) had injuries related to diving, 20 (13\%) had injuries from falls, 27 (18\%) had injuries sustained in sports/athletic competition, and 4 (3\%) sustained an SCI by other mechanisms. The injury breakdown is shown in Table 1.

Based on MRI findings, 23 people (55\%) with thoracic injuries and 70 people (64\%) with cervical injuries could not be enrolled in the trial based on imaging features. Of people with thoracic injuries in whom imaging findings prevented their inclusion, MR images from 10 people (24\%) exhibited an area of signal abnormality within the spinal cord with a rostral-caudal length of $>3 \mathrm{~cm}, 12$ studies $(29 \%)$ revealed a cavity structure not compatible with cell transplantation, and 1 study (2\%) showed syringomyelia. Of people with cervical injuries in whom imaging findings prevented their inclusion, MR images from 53 (48\%) exhibited an area of signal abnormality within the spinal cord with a rostral-caudal length of $>3 \mathrm{~cm}, 16$ studies $(15 \%)$ revealed a cavity structure not compatible with cell transplantation, and 1 study (1\%) showed syringomyelia. Imaging characteristics resulting in screening failure are given in Table 2.

Of all cases of thoracic injuries that failed screening, the average rostral-caudal lesion length was $50 \mathrm{~mm}$ (SD 41 $\mathrm{mm})$. Studies obtained in 11 people (35\%) with thoraciclevel SCI displayed associated tethering, $33(97 \%)$ revealed associated myelomalacia, 18 (58\%) showed an injury with cystic components, and 9 (36\%) demonstrated an injury with associated septations. Interquartile ranges for lesion length are also provided in Table 3.

Similarly, in all people with cervical injuries in whom screening failed to gain them access to the trial, the average sagittal lesion length was $34 \mathrm{~mm}$ (SD $21 \mathrm{~mm}$ ). Imaging studies from 32 people (36\%) exhibited an injury with associated tethering, 89 studies (100\%) showed an injury with associated myelomalacia, 73 studies (82\%) revealed an injury with cystic components, and 58 studies (75\%) displayed an injury with associated septations.

\section{Discussion}

In this study we provided an analysis of patients who did not meet the criteria for enrollment in our cell transplantation trial based on imaging characteristics. The cost and resources required for human cell transplantation trials require careful planning, and a better understanding of 
TABLE 3. Imaging characteristics

\begin{tabular}{lcc}
\hline \multirow{2}{*}{ Finding } & \multicolumn{2}{c}{ Level of Injury } \\
\cline { 2 - 3 } & Cervical & Thoracic \\
\hline Lesion rostral-caudal length, mm & & \\
\hline Mean (SD) & $34(21)$ & $50(41)$ \\
\hline Range & $6.3-108$ & $12-220$ \\
\hline Percentile & & \\
\hline 25th & 16 & 25 \\
\hline 50th & 31 & 38 \\
\hline 75th & 45 & 63 \\
\hline 95th & 84 & 171 \\
\hline Tethering (\%) & $32(36)$ & $11(35)$ \\
\hline Myelomalacia (\%) & $89(100)$ & $33(97)$ \\
\hline Cysts (\%) & $73(82)$ & $18(58)$ \\
\hline Septations (\%) & $58(75)$ & $9(36)$ \\
\hline
\end{tabular}

Percentages were calculated from the total number of people in whom the relevant finding could be assessed.

barriers to study inclusion will be of use in future studies. Of the over 400 people who were prescreened and the 160 who eventually submitted MRI data, just 8 were selected to undergo transplantation. We reviewed the MRI records of 152 applicants, who exhibited detailed imaging characteristics that excluded them from our study. As 93 individuals (61\%) were excluded on the basis of imaging criteria, our experience suggests that imaging characteristics posed the greatest barrier to enrollment for this particular study, which involved transplanting cells into a small, welldefined cystic cavity. Indeed, the average rostral-caudal extent of spinal cord signal abnormality was $34 \mathrm{~mm}$ in the cervical injury group and $50 \mathrm{~mm}$ in the thoracic injury group. These averages exceeded the criteria that we set as $3 \mathrm{~cm}$ (or $30 \mathrm{~mm}$ ) for our study inclusion (Fig. 1). Of note, these lengths were calculated from all applicants who did meet screening inclusion criteria and were not limited to those excluded only on the basis of imaging alone. This finding warrants careful consideration in the planning of future studies.

The rostral-caudal extent of signal abnormality and the cavity structure were the primary limiting characteristics in people who did not meet the criteria for enrollment. Sample cases are shown in Fig. 2. A large proportion of people (82\% in the cervical group and 58\% in the thoracic group) had visible cystic cavities, a well-studied phenomenon in chronic SCI. ${ }^{20}$ However, larger cavities, multiple septations, or loculated structures may inhibit successful intralesional grafting. Earlier studies have used $6 \mathrm{~cm}$ as a cutoff, ${ }^{8}$ but obliteration of cystic space following SCI is an important function of most graft models, ${ }^{25}$ and it may be easier accomplished with smaller cavities.

The limitations of MRI present challenges in the screening process. Image resolution, as well as the presence of spinal instrumentation artifact, made visualization of cystic changes difficult in a significant number of screened applicants. In some cases, MRI techniques, such as metal artifact suppression protocols, can be utilized to produce better quality imaging for evaluation of the spinal cord ar-
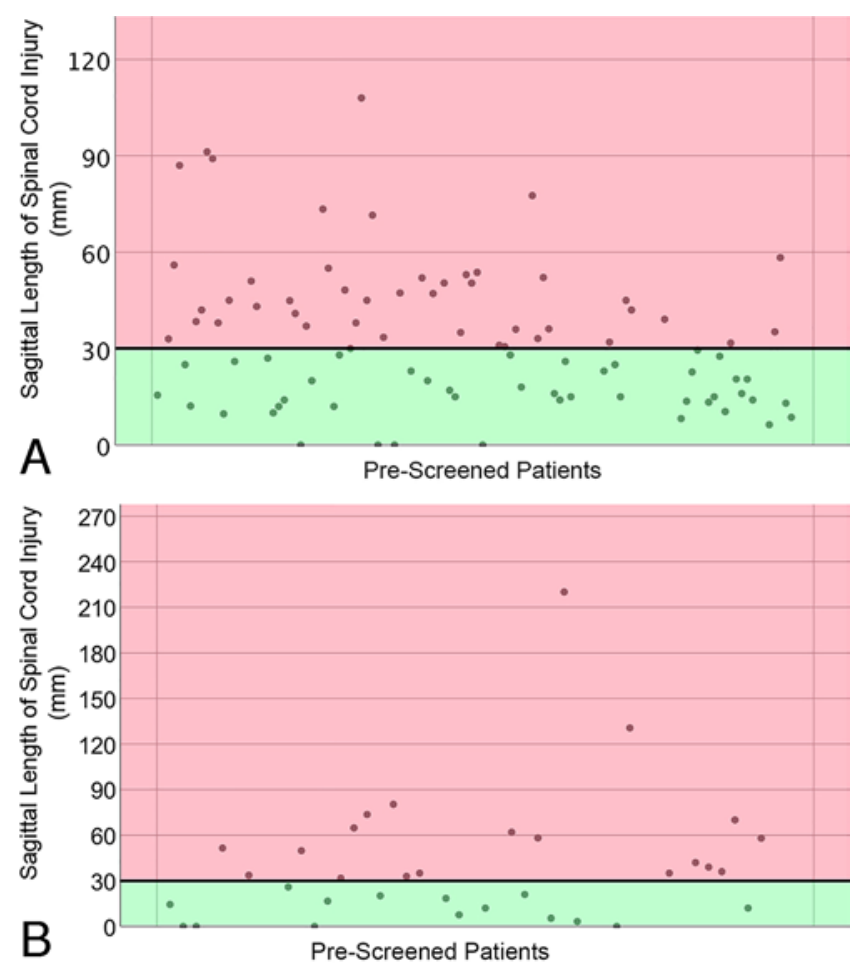

FIG. 1. Scatterplots illustrating sagittal length of spinal cord lesions among people with chronic cervical (A) and chronic thoracic (B) SCl. Applicants with MRI signal change $<30 \mathrm{~mm}$ but $>0$ could be considered for cell transplantation (green area). More people had sagittal signal change $>30 \mathrm{~mm}$ and were therefore eliminated from the trial in the screening process (red area).

chitecture. ${ }^{5,11,14}$ Due to the mechanisms of some SCIs, both anterior and posterior instrumentation may be required for stabilization. Metal-induced artifacts can significantly limit visualization of the spinal cord, despite artifact suppression techniques, and render the studies nondiagnostic. Unfortunately, potential participants with extensive hardware artifact may be screened out of cell transplantation trials for this inability to undergo spinal cord architecture assessment. There may be a role in future studies for additional imaging modalities such as diffusion tensor imaging and perfusion imaging to further define residual structure. ${ }^{713}$ However, as these modalities are not yet widely available, they can do little to aid in the screening process at this time. Regardless of which techniques are employed, our experience suggests that only a small subset of people with chronic SCI who are interested in participating in cellular transplantation clinical trials designed to bridge lesion cavities will qualify.

This study further underscores the importance of selection criteria in SCI trials. Enrolling participants comes with a high burden to both applicants and study sites, and more efficient study designs are needed in the field of SCI research. ${ }^{26}$ Fortunately, the screening process for our study incurred little cost to applicants, as they were required to submit records and images that had been obtained as part of routine care. However, the screening process did require substantial commitment from our institution. Future studies should appreciate the burdens of the screening process, which requires dedicated staff for correspondence with in- 

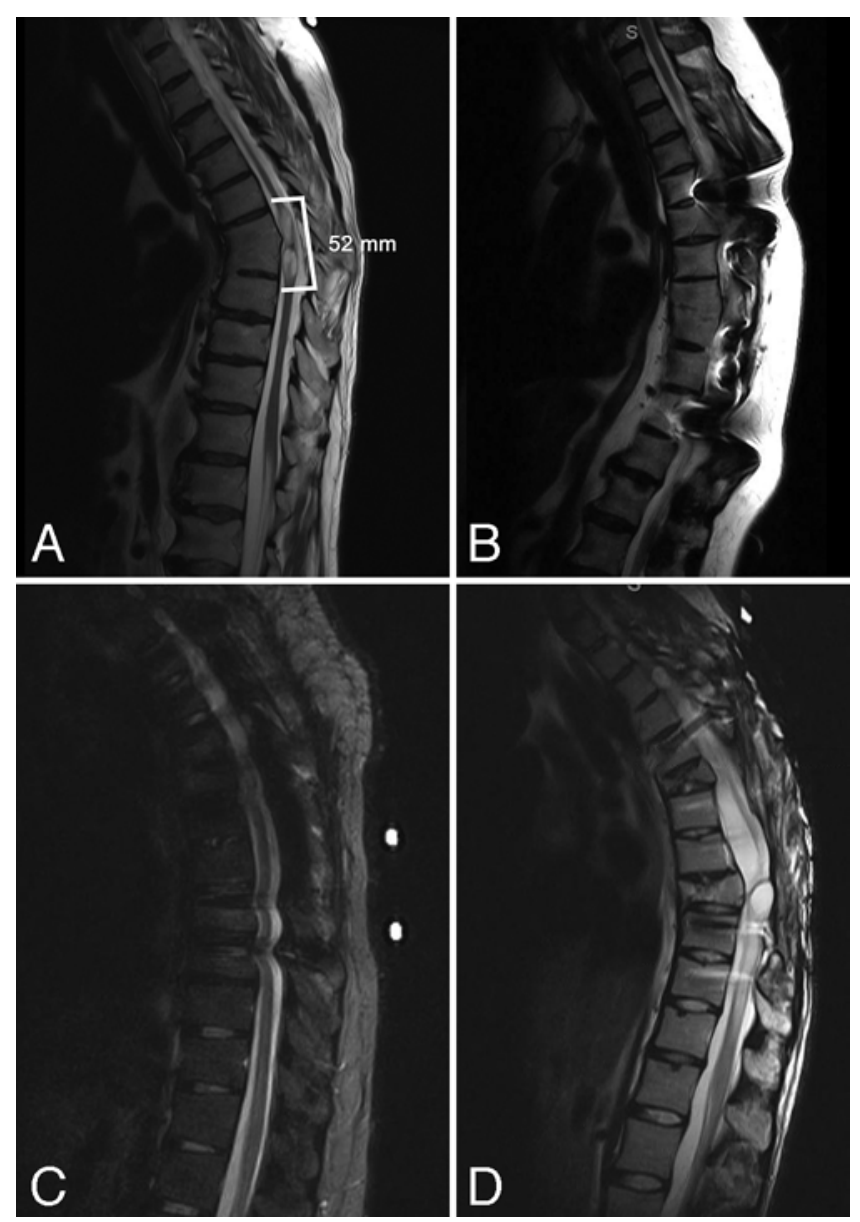

FIG. 2. Typical images from candidates excluded from the trial at the screening stage. A: MR image of a 57-year-old man who was involved in an MVA 30 years prior, with American Spinal Injury Association grade A $\mathrm{SCl}$. The sagittal length of the injury $(52 \mathrm{~mm})$ is too long for the individual to be considered for cell transplantation. Additionally, the presence of multiple septations and extensive myelomalacia make this person a poor transplant candidate. B: MR image of a 51-year-old woman involved in an MVA 21 years prior, with a complete $\mathrm{SCl}$ at the T7 vertebra. The structure of the cyst cannot be visualized due to artifact from hardware that was placed in her initial surgery. C: Sagittal image of a 27-year-old man who had been involved in an accident 4 years prior, demonstrating the absence of a cystic cavity that would be a necessary criterion for cell transplantation. D: Sagittal image of a 28 -year-old woman who had been involved in an MVA 3 years before, showing a complex cystic structure adjacent to the syrinx. There is also extensive myelomalacia rostral to the cyst. Any of these characteristics alone would preclude this candidate from inclusion in the trial.

terested applicants, and acquisition and review of screening documentation, including analysis of imaging data.

\section{Study Limitations}

This study was affected by inherent limitations of conventional MRI. The image quality of some of the MRI studies submitted was poor. Image quality is a significant barrier to screening and potential enrollment. Open MRI studies produce images that are often of lesser quality than traditional closed MRI. Traditionally, a 3-T MRI unit is able to produce better images than a $1.5-\mathrm{T}$ unit by creating a stronger signal-to-noise ratio. However, in postop- erative patients who have implanted hardware a 3-T MRI unit will produce more metal artifact and a 1.5-T imager is preferred. Availability of high-quality MRI studies may vary depending on the resources of the institution where patients have received their care. It is equally important for the technician acquiring the MR images to have an understanding of the clinical context (e.g., postsurgical patient with implanted hardware). Furthermore, an understanding of the optimal magnetic field strength and the appropriate MRI sequence protocols is essential for producing the best quality images.

Another limitation to recruitment of this trial was the inability of many applicants to submit recent images for evaluation. Some potential trial candidates only submitted images from the day of or immediately following their SCI, as some were unable to obtain or submit a recent MRI. The study did cover expenses for imaging for local applicants who met all other eligibility criteria. Current standards of care do not include annual imaging, or any imaging for that matter, unless there is a clinical indication. Others submitted imaging in a format that could not be viewed or with software with which structures could not be measured due to lack of calibration.

\section{Conclusions}

We present our experience in screening MRI studies of potential participants with chronic SCI for an intralesional cell transplantation clinical trial. Lesion length in chronic SCI was deemed an extremely important inclusion variable. The results help determine the feasibility of future clinical chronic SCI studies by helping investigators understand the large SCI population that they must have access to. Based on our trial criteria, only a fraction of applicants qualified, with many being excluded due to imaging characteristics. In trial planning, the workload of screening should be anticipated. If the number of selected candidates is likely to be low as in our case, care should be taken to temper expectations of the application process to reduce disappointment associated with screen failure.

\section{References}

1. Anderson KD, Guest JD, Dietrich WD, Bartlett Bunge M, Curiel R, Dididze M, et al: Safety of autologous human Schwann cell transplantation in subacute thoracic spinal cord injury. J Neurotrauma 34:2950-2963, 2017

2. Backe HA, Betz RR, Mesgarzadeh M, Beck T, Clancy M: Post-traumatic spinal cord cysts evaluated by magnetic resonance imaging. Paraplegia 29:607-612, 1991

3. Bastidas J, Athauda G, De La Cruz G, Chan WM, Golshani R, Berrocal Y, et al: Human Schwann cells exhibit long-term cell survival, are not tumorigenic and promote repair when transplanted into the contused spinal cord. Glia 65:12781301, 2017

4. Bozzo A, Marcoux J, Radhakrishna M, Pelletier J, Goulet B: The role of magnetic resonance imaging in the management of acute spinal cord injury. J Neurotrauma 28:1401-1411, 2011

5. Cha JG, Jin W, Lee MH, Kim DH, Park JS, Shin WH, et al: Reducing metallic artifacts in postoperative spinal imaging: usefulness of IDEAL contrast-enhanced T1- and T2-weighted MR imaging - phantom and clinical studies. Radiology 259:885-893, 2011 
6. Curtis E, Martin JR, Gabel B, Sidhu N, Rzesiewicz TK, Mandeville R, et al: A first-in-human, phase I study of neural stem cell transplantation for chronic spinal cord injury. Cell Stem Cell 22:941-950.e6, 2018

7. Ellingson BM, Salamon N, Holly LT: Imaging techniques in spinal cord injury. World Neurosurg 82:1351-1358, 2014

8. Falci S, Holtz A, Akesson E, Azizi M, Ertzgaard P, Hultling C, et al: Obliteration of a posttraumatic spinal cord cyst with solid human embryonic spinal cord grafts: first clinical attempt. J Neurotrauma 14:875-884, 1997

9. Flanders AE, Spettell CM, Friedman DP, Marino RJ, Herbison GJ: The relationship between the functional abilities of patients with cervical spinal cord injury and the severity of damage revealed by MR imaging. AJNR Am J Neuroradiol 20:926-934, 1999

10. Ghobrial GM, Anderson KD, Dididze M, Martinez-Barrizonte J, Sunn GH, Gant KL, et al: Human neural stem cell transplantation in chronic cervical spinal cord injury: functional outcomes at 12 months in a phase II clinical trial. Neurosurgery 64 (CN_suppl_1):87-91, 2017

11. Jungmann PM, Agten CA, Pfirrmann CW, Sutter R: Advances in MRI around metal. J Magn Reson Imaging 46:972-991, 2017

12. Kirshblum SC, Waring W, Biering-Sorensen F, Burns SP, Johansen M, Schmidt-Read M, et al: Reference for the 2011 revision of the International Standards for Neurological Classification of Spinal Cord Injury. J Spinal Cord Med 34:547554,2011

13. Koskinen EA, Hakulinen U, Brander AE, Luoto TM, Ylinen A, Ohman JE: Clinical correlates of cerebral diffusion tensor imaging findings in chronic traumatic spinal cord injury. Spinal Cord 52:202-208, 2014

14. Lee YH, Lim D, Kim E, Kim S, Song HT, Suh JS: Usefulness of slice encoding for metal artifact correction (SEMAC) for reducing metallic artifacts in 3-T MRI. Magn Reson Imaging 31:703-706, 2013

15. Levi AD, Anderson KD, Okonkwo DO, Park P, Bryce TN, Kurpad SN, et al: Clinical outcomes from a multi-center study of human neural stem cell transplantation in chronic cervical spinal cord injury. J Neurotrauma [epub ahead of print], 2018

16. Ma VY, Chan L, Carruthers KJ: Incidence, prevalence, costs, and impact on disability of common conditions requiring rehabilitation in the United States: stroke, spinal cord injury, traumatic brain injury, multiple sclerosis, osteoarthritis, rheumatoid arthritis, limb loss, and back pain. Arch Phys Med Rehabil 95:986-995, 995.e1, 2014

17. Nagoshi N, Khazaei M, Ahlfors JE, Ahuja CS, Nori S, Wang $\mathrm{J}$, et al: Human spinal oligodendrogenic neural progenitor cells promote functional recovery after spinal cord injury by axonal remyelination and tissue sparing. Stem Cells Transl Med 7:806-818, 2018

18. Ning GZ, Song WY, Xu H, Zhu RS, Wu QL, Wu Y, et al: Bone marrow mesenchymal stem cells stimulated with lowintensity pulsed ultrasound: better choice of transplantation treatment for spinal cord injury: treatment for SCI by LIPUSBMSCs transplantation. CNS Neurosci Ther [epub ahead of print], 2018

19. NSCISC: Spinal cord injury (SCI) 2016 facts and figures at a glance. J Spinal Cord Med 39:493-494, 2016

20. Quencer RM, Bunge RP: The injured spinal cord: imag- ing, histopathologic clinical correlates, and basic science approaches to enhancing neural function after spinal cord injury. Spine (Phila Pa 1976) 21:2064-2066, 1996

21. Quencer RM, Sheldon JJ, Post MJ, Diaz RD, Montalvo BM, Green BA, et al: MRI of the chronically injured cervical spinal cord. AJR Am J Roentgenol 147:125-132, 1986

22. Sett $P$, Crockard HA: The value of magnetic resonance imaging (MRI) in the follow-up management of spinal injury. Paraplegia 29:396-410, 1991

23. Silberstein M, Hennessy O: Implications of focal spinal cord lesions following trauma: evaluation with magnetic resonance imaging. Paraplegia 31:160-167, 1993

24. Squier MV, Lehr RP: Post-traumatic syringomyelia. J Neurol Neurosurg Psychiatry 57:1095-1098, 1994

25. Thuret S, Moon LD, Gage FH: Therapeutic interventions after spinal cord injury. Nat Rev Neurosci 7:628-643, 2006

26. Tuszynski MH, Steeves JD, Fawcett JW, Lammertse D, Kalichman M, Rask C, et al: Guidelines for the conduct of clinical trials for spinal cord injury as developed by the ICCP Panel: clinical trial inclusion/exclusion criteria and ethics. Spinal Cord 45:222-231, 2007

27. Wang D, Bodley R, Sett P, Gardner B, Frankel H: A clinical magnetic resonance imaging study of the traumatised spinal cord more than 20 years following injury. Paraplegia 34:6581, 1996

28. Yamashita Y, Takahashi M, Matsuno Y, Sakamoto Y, Oguni T, Sakae T, et al: Chronic injuries of the spinal cord: assessment with MR imaging. Radiology 175:849-854, 1990

\section{Disclosures}

Dr. Guest serves on the Grants Working Group of the California Institute for Regenerative Medicine and as a liaison to the Asterias Biotherapeutics OPC-1 clinical trial. Additionally, he serves as an advisor to the FDA for SCI therapeutics. Ms. Khan holds equity in AssureImmune Cord Blood Bank. Dr. Levi receives teaching honorarium from the American Association of Neurological Surgeons and grant support from the Department of Defense and the National Institutes of Health (NIH-NINDS).

\section{Author Contributions}

Conception and design: Levi, Gant. Acquisition of data: Burks, Gant, Jamshidi, Cox. Analysis and interpretation of data: Levi. Drafting the article: Burks, Gant, Jamshidi, Cox. Critically revising the article: Levi, Burks, Gant, Guest, Anderson, Dietrich, Bunge, Green, Khan, Pearse, Saraf-Lavi. Reviewed submitted version of manuscript: all authors. Approved the final version of the manuscript on behalf of all authors: Levi. Statistical analysis: Burks.

\section{Supplemental Information \\ Videos}

Video Abstract. https://vimeo.com/315448816.

\section{Correspondence}

Allan D. Levi: University of Miami Miller School of Medicine, Miami,FL.alevi@med.miami.edu. 